\title{
Early interventions to promote work participation in people with regional musculoskeletal pain: a systematic review and meta-analysis
}

Clinical Rehabilitation 2017, Vol. 3I(II) 1466-148I

(C) The Author(s) 2017

Reprints and permissions: sagepub.co.uk/journalsPermissions.nav DOI: $10.1177 / 0269215517699976$ journals.sagepub.com/home/cre @SAGE

\section{Andy Cochrane', Niamh M Higgins', Oliver FitzGerald ${ }^{2}$, Pamela Gallagher ${ }^{3}$, Jennifer Ashton ${ }^{4}$, Oriel Corcoran ${ }^{5}$ and Deirdre Desmond'}

\begin{abstract}
Objectives: To determine the effectiveness of early multidisciplinary interventions in promoting work participation and reducing work absence in adults with regional musculoskeletal pain.

Data sources: Seven databases (CENTRAL, CINAHL, EMBASE, MEDLINE, Scopus, OT Seeker, PEDro; 1990 to December 2016) were searched for eligible studies.

Review methods: Trials were included if they reported on work-based outcomes for participants experiencing difficulties at work or $\leqslant$ three months' sick leave. Interventions had to include two or more elements of the biopsychosocial model delivered as a coordinated programme. Quality was assessed using the GRADE criteria. Results were analysed by hazard ratios for return to work data; continuous outcomes were analysed as standardised mean difference with $95 \%$ confidence intervals.

Results: A total of 20 randomized controlled trials, with 16,319 participants were included; the interventions were grouped according to their main components for meta-analyses. At 12-months follow-up, moderate quality evidence suggests that programmes involving a stepped care approach (four studies) were more effective than the comparisons in promoting return to work (hazard ratio (HR) I.29 ( $95 \%$ confidence interval $(\mathrm{Cl}) \mathrm{I} .03$ to I.6I), $p=0.03$ ), whereas case management (two studies) was not (HR 0.92 (95\% Cl 0.69 to I.24), $p=0.59)$. Analyses suggested limited effectiveness in reducing sickness absences, in pain reduction or functional improvement across the intervention categories.

Conclusion: There is uncertainty as to the effectiveness of early multicomponent interventions owing to the clinical heterogeneity and varying health and social insurance systems across the trials.
\end{abstract}

'Department of Psychology, Maynooth University, Maynooth, Ireland

${ }^{2}$ School of Medicine and Medical Sciences, University College

Dublin, Dublin, Ireland

${ }^{3}$ School of Nursing and Human Sciences, Dublin City

University, Dublin, Ireland

${ }^{4}$ Physiotherapy Services, Beaumont Hospital, Dublin, Ireland
${ }^{5}$ Rheumatology Services, Waterford Regional Hospital, Waterford, Ireland

Corresponding author:

Andy Cochrane, Department of Psychology, National

University of Ireland Maynooth, John Hume Building,

Maynooth, Ireland.

Email: andy.cochrane@nuim.ie 


\section{Keywords}

Musculoskeletal disorders, pain, return to work, vocational rehabilitation

Received: I 5 July 2016; accepted: 25 February 2017

\section{Introduction}

Many adults experience symptoms of musculoskeletal disorders at some time during their working life. ${ }^{1}$ While the majority of these episodes are self-limiting, musculoskeletal disorders (MSDs) remain the leading cause of temporary absences from work and permanent work disability across Europe. ${ }^{2}$ Sickness absence and lost work productivity costs the European Union an estimated 240 billion euro annually. ${ }^{3}$ Musculoskeletal conditions account for around $20 \%$ of claims for long-term incapacity benefits in the $\mathrm{UK},{ }^{4}$ and those with a persisting work disability are at greater risk of poor mental health and decreased quality of life. ${ }^{5}$

Work instability and/or disability is not just the consequence of a clinical impairment, but rather is influenced by a number of inter-related factors, including individual psychosocial characteristics, the workplace environment and the social protection system. ${ }^{6}$ Acknowledgement of the multicausal nature of work absence and disability suggests that programmes that address the range of relevant biopsychosocial factors might be most effective in reducing sickness absence and promoting return to work. ${ }^{7}$ While there have been a number of systematic reviews on the impact of interventions on work outcomes, they have typically involved single diagnostic groups and/or targeted patients who have chronic pain, with inconsistent findings. ${ }^{8-10}$

The lack of robust consistent findings may be explained, in part, by a lack of clarity regarding the optimum timing of intervention. ${ }^{11}$ Evidence suggests that the probability of sustained return to work is reduced the longer an individual is out of work. ${ }^{12}$ Early intervention has the potential to ensure that people are appropriately managed and supported before work absence becomes long-term. ${ }^{13}$ This review differs, therefore, from previous reviews by focusing specifically on multicomponent, biopsychosocial interventions that recruit participants in the first three months of sick leave. Until recently, most evidence on work-related outcomes ${ }^{7}$ has focused on low back pain; this review will include a wider range of pain disorders that are of importance (e.g. shoulder/neck/forearm pain and knee pain) in relation to work disability. ${ }^{14}$

The review objective was to examine the effectiveness of multicomponent interventions, delivered early in the onset of difficulties at work or work absence, for promoting work participation and reducing the duration of sickness absence for people experiencing musculoskeletal pain.

\section{Method}

The systematic review was conducted in accordance with the Preferred Reporting Items for Systematic Reviews and Meta-Analyses (PRISMA) statement. ${ }^{15}$ The review was registered with PROSPERO (International Prospective Register of Systematic Reviews: registration number: CRD42015019351) prior to the literature search.

\section{Search strategy}

We conducted a systematic search of electronic databases (CENTRAL, MEDLINE, CINAHL, EMBASE, SCOPUS, PEDro, OT Seeker) between 1990 and 2016 inclusive. See Appendix 1, available online, for the MEDLINE search strategy. Searches were limited to literature published in English. We also screened existing systematic reviews and the reference lists of other relevant articles to identify potentially eligible trials. Titles and abstracts were screened for eligibility; those deemed immediately irrelevant were discarded. Two review authors independently reviewed the full text of the remaining studies; any disagreement was resolved by discussion and consensus. We 
contacted study authors for further information where the eligibility of the study was unclear.

\section{Eligibility criteria}

Trial design. Randomized controlled trials (RCTs), cluster randomized trials and quasi-randomized controlled trials that compared an early intervention with 'treatment as usual', 'wait list' or an alternative active intervention.

Population. People aged 18 or over with musculoskeletal pain (e.g. back pain, shoulder/neck/forearm pain and knee pain) who met the following criteria:

- $80 \%$ or more of the sample were in paid employment at the time of recruitment;

- three months or less of sickness absence from work, related to musculoskeletal pain, during the previous year - if the sample involved participants with longer periods of sick leave, the study was included if less than $20 \%$ of the sample had more than three months sick leave.

Trials focused on patients with inflammatory conditions (i.e. rheumatoid arthritis, ankylosing spondylitis, sero-negative arthritis, connective tissue diseases and psoriatic arthritis) were excluded. We considered trials with mixed populations if the inflammatory conditions comprised less than $10 \%$ of the overall sample.

Intervention. Trials that involved two or more different components from the biopsychosocial model delivered as an integrated programme by a multidisciplinary team or single health professional were included. In the absence of fixed or standard components of the biopsychosocial model, we adopted the criterion from an earlier review ${ }^{16}$ and included trials where the intervention comprised a physical (bio-) component and at least one psychosocial element.

- Physical/bio: The participant was assessed by physician, physiotherapist or other health professional for causes of their pain and received exercise/physical therapy if indicated.
- Psychological, for example: Education, selfmanagement training, coping with pain and unhelpful beliefs, counselling and cognitive behavioural approaches.

- Social/occupational, for example: Workplace assessment and adaptations or barriers to work, development of communication and problemsolving skills.

The intervention could be of any intensity, and delivered to individuals or groups in a variety of settings, including hospital, community and the workplace. Trials of primary prevention for healthy workers and of surgical interventions were excluded. Control groups consisted of: (1) the usual treatment available in the trial location; (2) wait-list; or (3) active intervention arms.

Outcomes. Trials must have measured one of the following work outcomes: (1) duration of sick leave, or (2) time to return to work. Secondary outcomes included: pain; disability; psychological functioning; quality of life; fatigue; and adverse effects. We planned to consider work productivity, presenteeism and healthcare utilisation if a sufficient number of trials included these as outcomes. Studies of cost effectiveness were included if conducted alongside or subsequent to a trial that met the inclusion criteria. We included trials that reported outcomes for short-term (e.g. 3-6 months) and long-term follow-up (e.g. 12 months or longer).

\section{Risk of bias assessment}

Methodological risk of bias was assessed in accordance with Cochrane guidelines. ${ }^{17}$ The six main domains of the risk of bias tool and the following other potential sources of bias were assessed: (1) baseline comparability of groups; (2) compliance with intervention; and (3) use of co-interventions. Each item was judged separately as being at high, low, or unclear risk of bias. ${ }^{17}$ Studies were assigned a low quality (low risk of bias on four or less items); moderate quality (low risk of bias on 5-7 items) or high quality rating (low risk of bias on eight or more items). Two reviewers independently assessed the risk of bias of included studies; any 
disagreements were resolved by discussion and consensus.

\section{Quality of the evidence}

Two reviewers independently assessed the quality of the evidence for each outcome using the GRADE (Grades of Recommendation, Assessment, Development and Evaluation) criteria ${ }^{17}$ for each of the following parameters: Risk of bias, inconsistency, imprecision, indirectness and publication bias. A rating of 'high quality' evidence was downgraded by one level for serious concerns, and by two levels for very serious concerns.

\section{Data extraction and synthesis of results}

Data were extracted independently by two reviewers including: Participants, diagnosis and setting; intervention characteristics (including timing and intensity); comparison group details; assessment timeframes; outcomes. Study authors were contacted to clarify methodological components and/ or access unpublished outcome data (for example means and standard deviations). Where necessary, methods described in the Cochrane Handbook were used to convert study results to the required format or to impute missing standard deviations. ${ }^{17}$ Where no estimates were possible using the methods outlined, the data were not used. Intentionto-treat (ITT) analyses were used when available. In trials where there were multiple groups from the same study (e.g. two active arms and a control group), we followed the approaches outlined in the Cochrane Handbook ${ }^{17}$ to either combine groups or conduct pair-wise comparisons as long as both active arms met our inclusion criteria.

Meta-analysis was conducted where homogeneity was sufficient in terms of the main components of the intervention, outcome domains and followup time point. Where time to return to work data were reported as hazard ratios, we obtained estimates of log hazard ratios and standard errors from the hazard regression models and study results were combined using the generic inverse-variance method. The duration of sickness absence days was measured for different time spans in the included trials; therefore, we used standardised mean differences (SMDs) with 95\% confidence intervals (CIs) and the random effects method to pool results. We also planned to use SMDs for other continuous variables (e.g. pain intensity and disability) as these were measured using different scales across trials. The degree of statistical heterogeneity was assessed by examining the Chi-squared test and the $I^{2}$ statistic. An $I^{2}$ value of $50 \%$ or more was considered to represent substantial heterogeneity. All analyses were calculated using Review Manager 5.3. ${ }^{18}$

\section{Results}

Figure 1 summarises the screening and selection process; the search resulted in 10,871 studies once duplicates were removed. We obtained the full text of 328 articles; twenty separate trials met our inclusion criteria.

\section{Trial characteristics}

Nineteen of the included studies were RCTs, one was a cluster RCT. The majority of the studies were conducted in Europe $(n=17)$, three were from Canada. Study characteristics are presented in Table 1. A very large-scale study from Spain ${ }^{19}$ recruited 13,077 participants; the sample sizes of the remaining studies ranged from 54 to 466 . The combined total was 16,319 participants, with mean age of 42.8 years (range $32-51$ ), and $54.27 \%$ were female. The majority of participants were on sick leave at the time of inclusion into the trials.

The interventions were conducted in various settings and there was considerable variation in the components employed (Table 1). The interventions were grouped into categories according to their main components as described in the publication and data, where available, were pooled for these groupings.

1. Back school programmes. ${ }^{20,21}$

2. Case-manager-led programmes. ${ }^{22-25}$

3. A focus on increasing physical activity in combination with multidisciplinary input. ${ }^{26-29}$ 


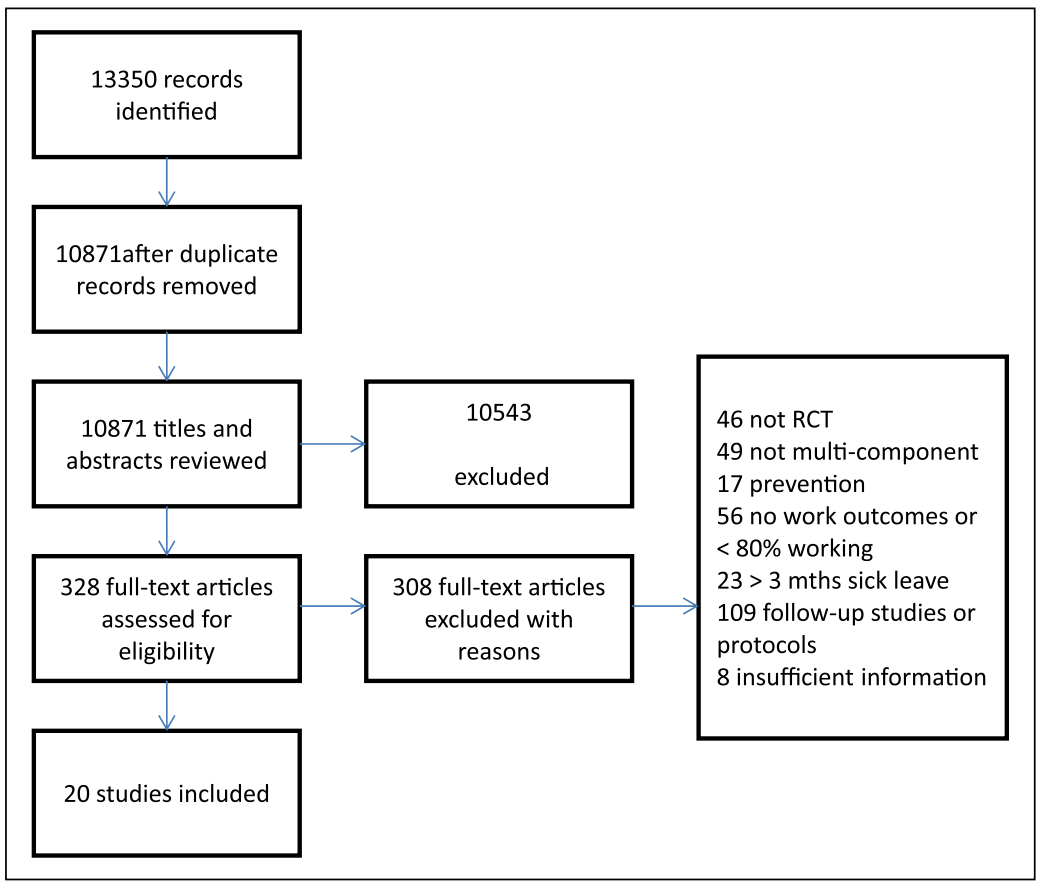

Figure I. Flowchart of screened, excluded and included studies. RCT: randomized controlled trial.

4. A psychosocial intervention; ${ }^{30,32}$ psychosocial in combination with exercise; ${ }^{31.33}$ workplace; ${ }^{34}$ or conventional clinical management. ${ }^{35}$

5. Stepped care approaches: (a) protocol-based rheumatologist-led clinical management with three levels; ${ }^{19}$ (b) the same rheumatologist-led clinical management supplemented with cognitive behavioural therapy; ${ }^{36}$ (c) occupational intervention followed by a clinical intervention (the Sherbrooke Model); ${ }^{37}$ (d) work assessment and adjustments directly after enrolment followed by graded activity for participants who had not returned to work after eight weeks. ${ }^{38}$

Five studies employed multiple groups (e.g. two active arms and a control group); only the arms that met the criteria for two or more components from the biopsychosocial model were used as the intervention group. Pair wise comparisons were used to include both active arms from two studies; ${ }^{20,27}$ in two studies two arms that did not meet the criterion were combined as a control group ${ }^{29,33}$ using the recommended statistical adjustments to sample sizes; ${ }^{17}$ in one study only data for a combined intervention (workplace and graded activity) was used in the analyses. ${ }^{38}$

\section{Quality of the evidence}

The studies were judged to be at low risk of bias for between three and eight of the nine categories, although in some cases there was insufficient information to make a judgement. Few studies provided adequate information relating to how treatment fidelity, compliance with the intervention and use of additional healthcare resources/co-interventions were monitored. Only one trial was rated as being of high quality, ${ }^{28} 11$ were rated as of moderate quality and eight of low quality, primarily owing to insufficient detail in the articles (see supplementary data available online). According to the GRADE assessment, the evidence was of very low to moderate quality primarily owing to risk of bias 


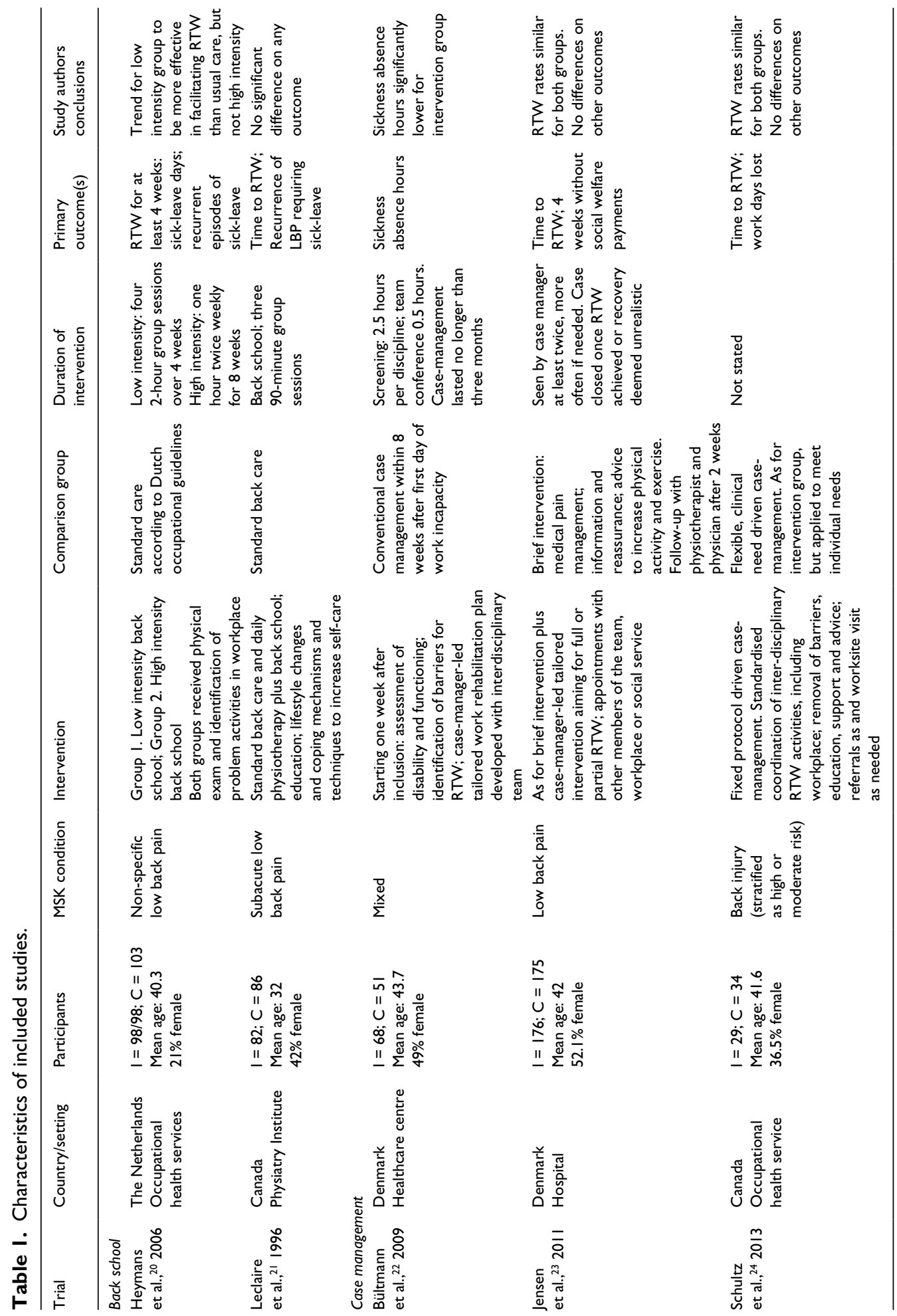




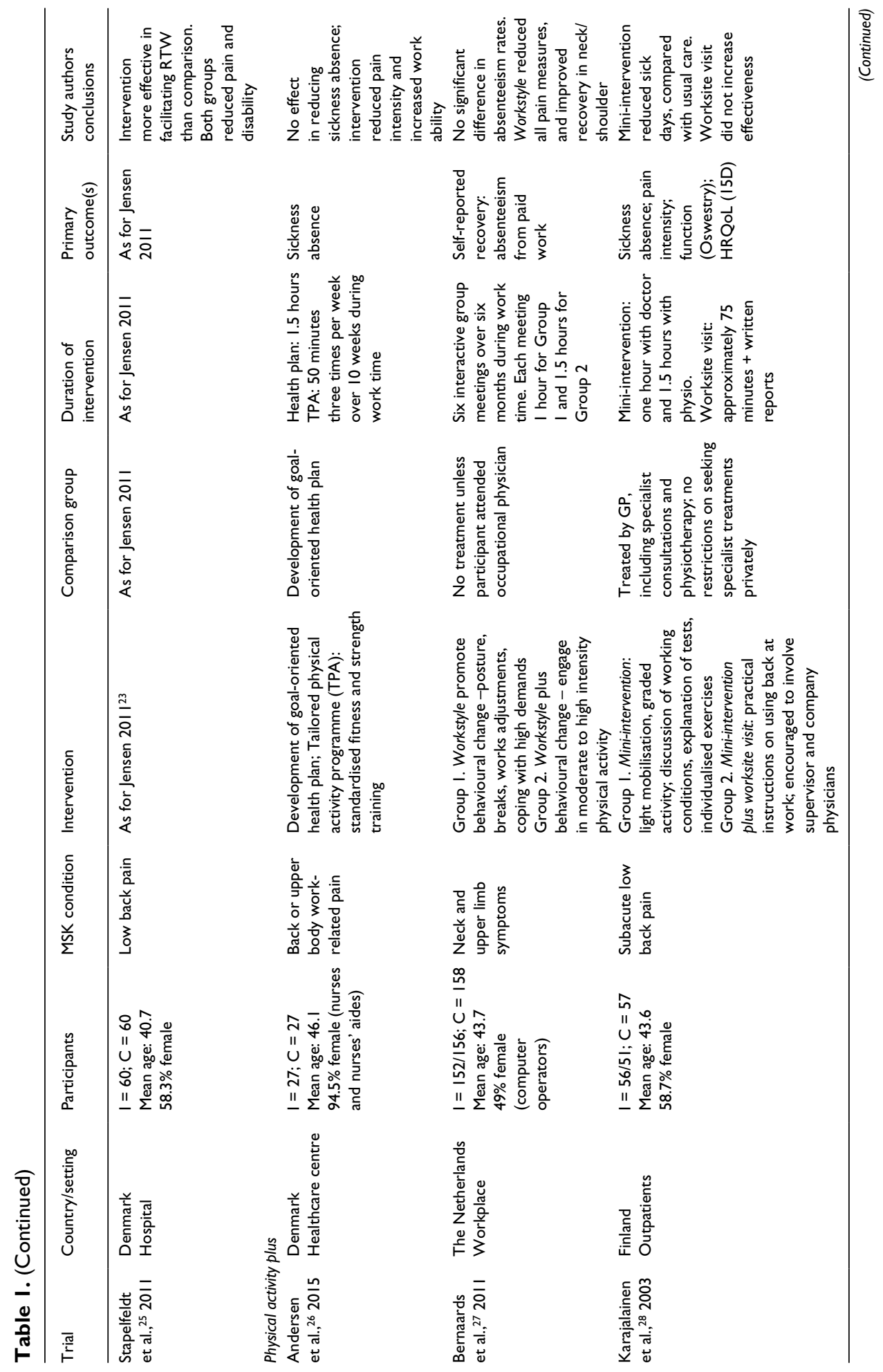




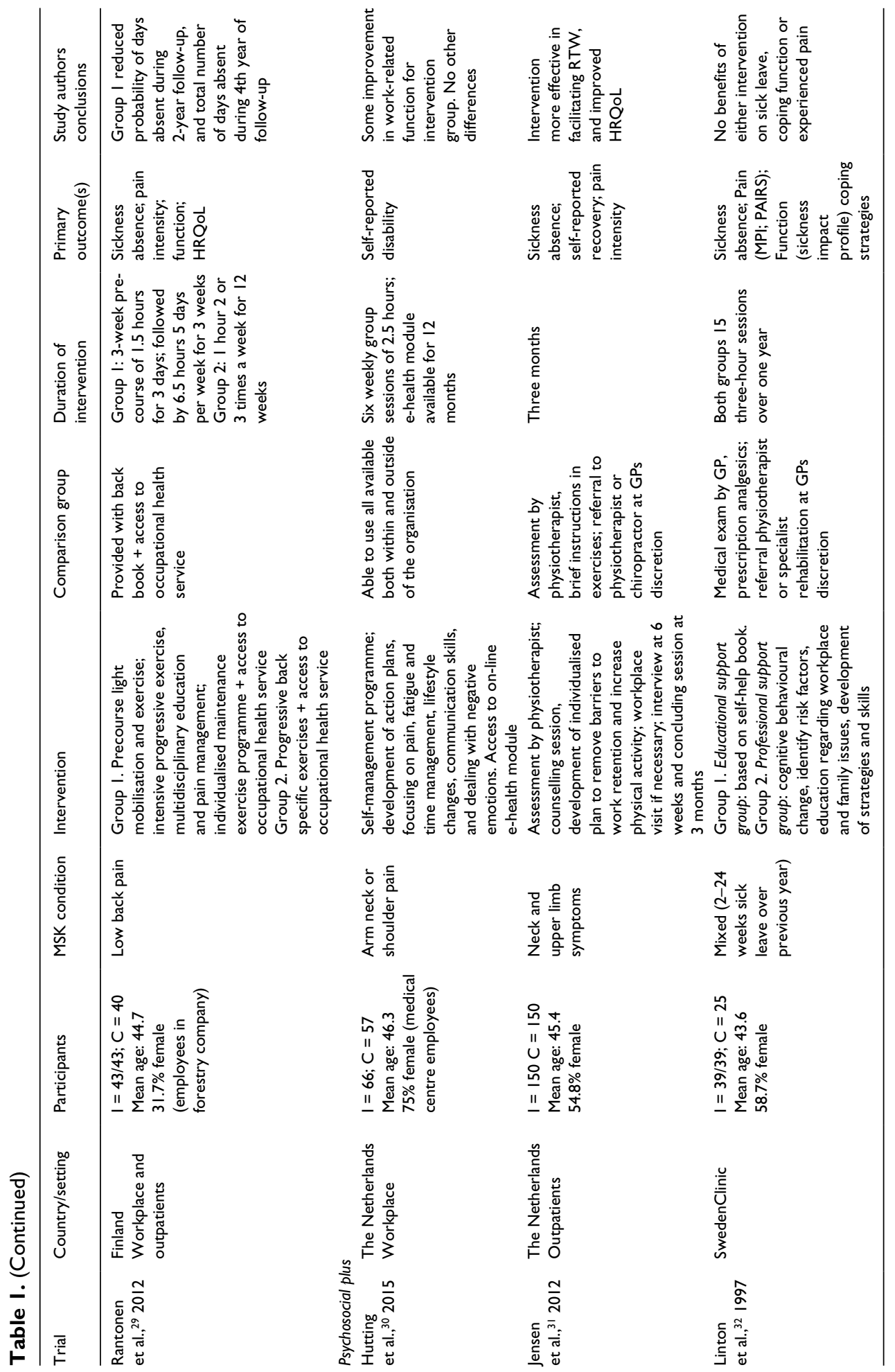




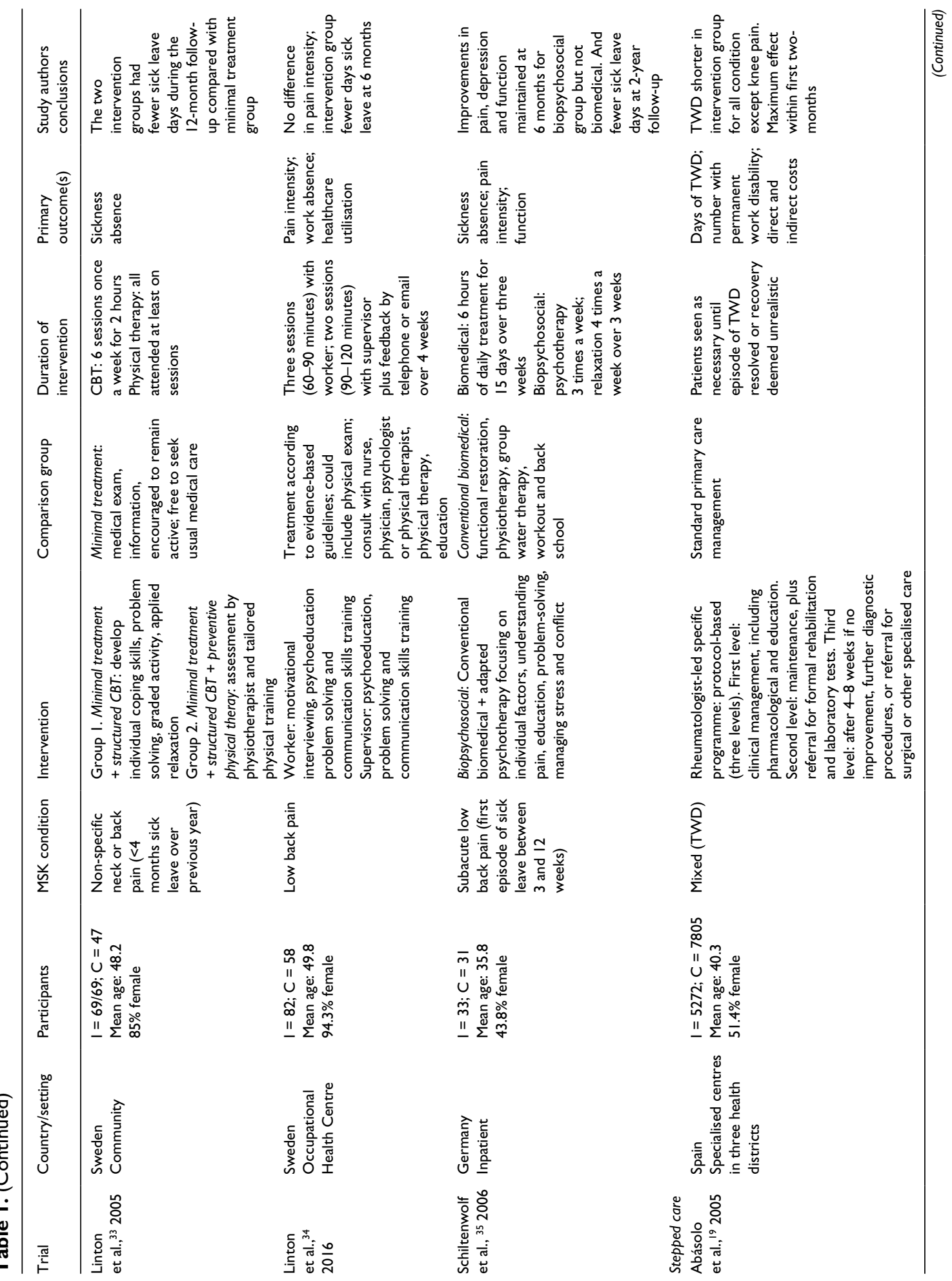




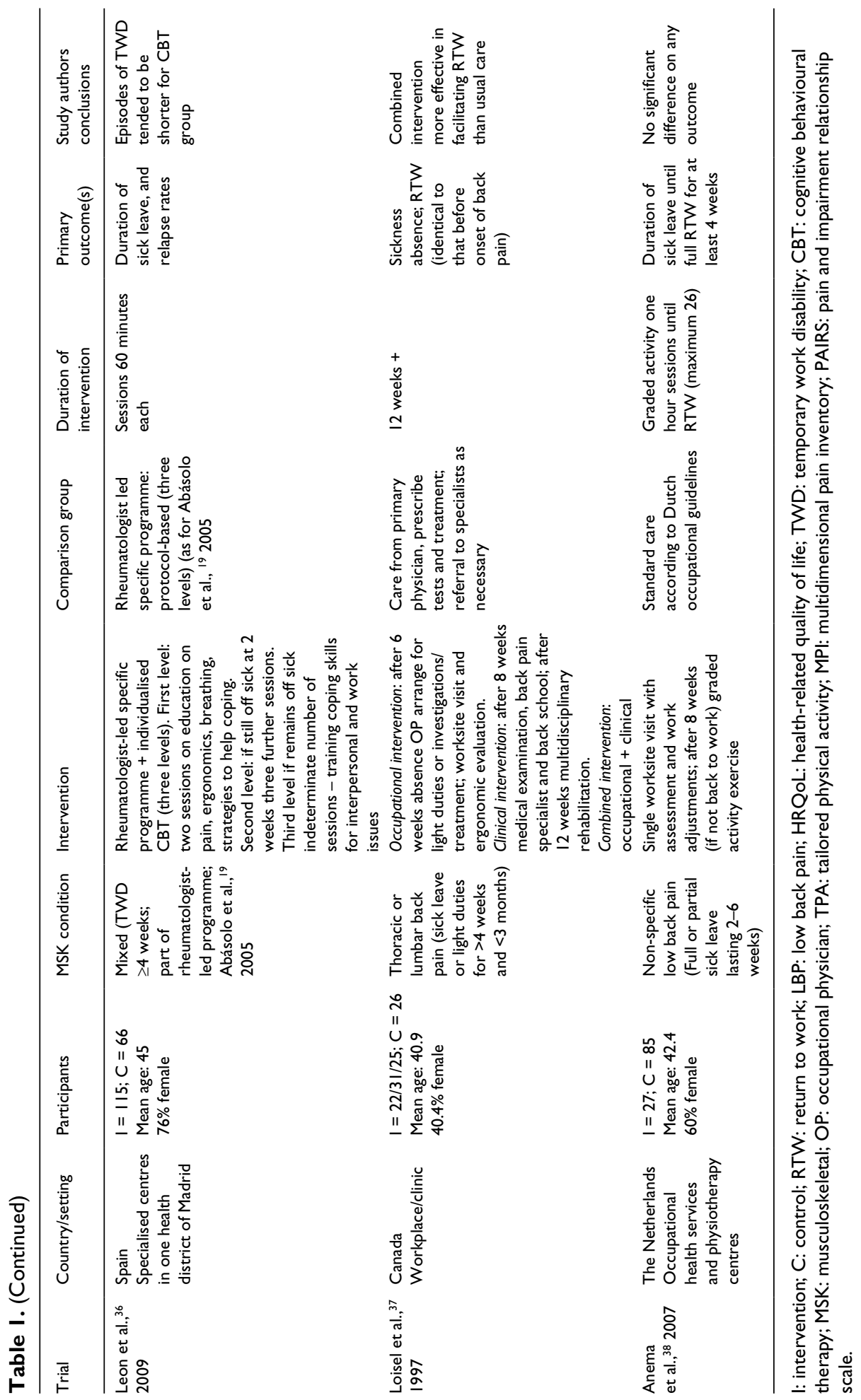


and imprecise results because of small sample size. Table 2 presents an overview of these judgements.

\section{Return to work}

The criteria used to define return to work varied, and included: (1) the cessation of temporary disability payments; $;^{19,33,36}(2)$ return to work identical to that before the onset of musculoskeletal (MSK) pain; ${ }^{37}$ (3) return to previous or equal work for at least four weeks. ${ }^{20,23,25,38}$

Hazard ratios for return to work were available for two studies at 3-6 months ${ }^{20,31}$ and six studies for 12-month follow-up. ${ }^{19,23,25,36,37,38}$ One trial of moderate quality ${ }^{31}$ found that a counselling-based intervention was more effective than usual care at three-month follow-up (hazard ratio (HR) 2.57, $95 \%$ CI 1.98 to $3.34 ; p=0.0001$ ). Four trials $19,36,37,38$ of low quality examined the effects of stepped care at 12 months, and the pooled data suggest that stepped care probably facilitates return to work more effectively than the comparison interventions (HR $1.29,95 \%$ CI 1.03 to $1.61 ; p=0.03, I^{2}=50 \%$ ). The moderate-quality pooled data for case management ${ }^{23,25}$ indicated that the intervention may make little or no difference above that of the comparison intervention (HR 0.92, 95\% CI 0.69, 1.24; $p=0.59, I^{2}=36 \%$ ). Two studies ${ }^{21,24}$ provided return to work data as a percentage of those back to work at 12 months, with no difference between intervention and control groups ( $p$ 's 0.61 and 0.14 , respectively). (See Table 2 and Supplementary Data for forest plots for all analyses.)

\section{Sickness absence}

Sickness absence, measured in days, was obtained from the records kept by social security and health insurance systems in ten studies, ${ }^{19,21-25,31,32,35,36}$ five accessed data through company records/ occupational health $20,27,29,37,38$ and five used selfreported data. ${ }^{26,28,30,33,34}$ Only 13 studies presented sickness absence data that could be used in the analyses. At a 3-6 month follow-up, low- to moderate-quality evidence revealed no difference for the psycho-social interventions ${ }^{30,34}$ case management ${ }^{24}$ nor back school (high and low intensity). ${ }^{20}$
For sickness absence rates at longer term followup, we found very low to moderate quality evidence of little or no difference above that of the comparison group across the intervention categories. ${ }^{19,22,23,24,27,29,30,35,36,37}$ Statistical heterogeneity was generally high with $I^{2}$ values ranging from $27 \%$ to $97 \%$.

Four studies ${ }^{19,20,21,36}$ reported on the number and duration of recurrent episodes of sickness absences related to musculoskeletal pain. The large-scale study conducted in Spain ${ }^{19}$ reported that a quarter of participants had more than one episode of temporary work disability during the follow-up period, with no difference between the intervention and control groups in the number of episodes per participant, although the duration of the episodes was shorter for the intervention group compared with the control (mean 25.33 days vs. 43.33 days, $p<$ 0.0001). Similar effects were reported for the other rheumatology-led study with added cognitive behavioural therapy component, ${ }^{36}$ the intervention group experienced shorter episodes of temporary work disability relative to the control group (mean 63.69 days vs. 197.62 days, $p=0.002$ ). The remaining two studies reported no differences between the intervention and comparison groups for recurrence rates or the duration of these episodes..$^{20,21}$

\section{Pain intensity and disability}

Three studies ${ }^{20,31,35}$ reported on pain intensity and disability at 3-6 months, and three $\mathrm{e}^{21,26,34}$ on pain only. At longer-term follow-up, eight studies $^{20-22,24,28,29,31,32}$ reported on both pain and disability, and one ${ }^{27}$ on pain only. There was no consistent evidence that any of the intervention categories had an effect on either pain intensity or disability above that of the comparison for either follow-up period.

\section{Early intervention and cost savings}

Eight studies collected direct health costs and indirect work- and benefits-related costs, and the majority adopted a human capital approach when estimating productivity loss. Methodological differences in terms of the interventions, health systems and the types of economic analyses make it 


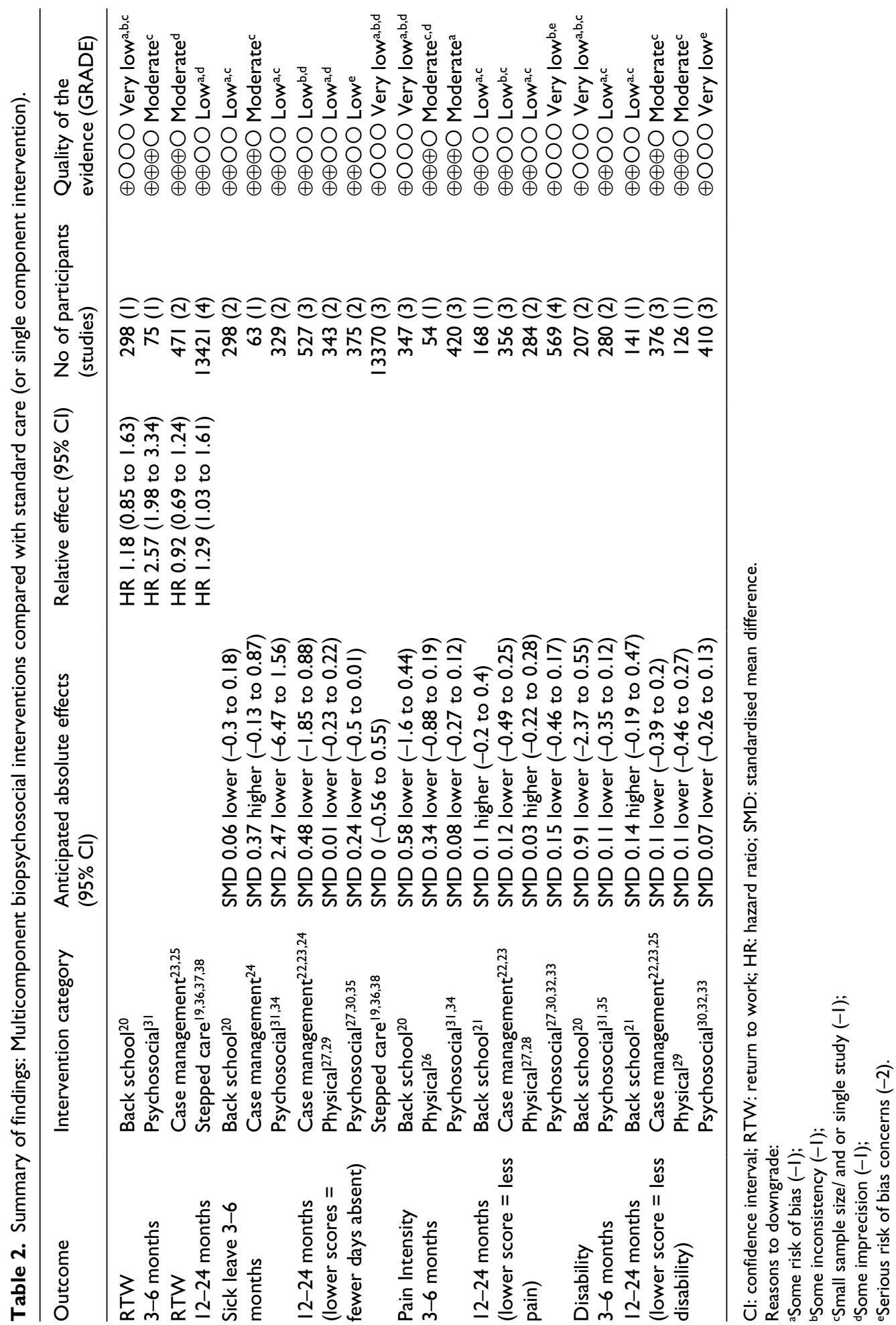


difficult to make direct comparisons across the trials. Three trials reported cost savings in health service costs and limiting productivity losses $19,22.36$ and also by reducing the number of patients transitioning to long-term disability. ${ }^{18}$ For example, every dollar invested produced savings of between US $\$ 4$ and US\$11 in the two Spanish studies 36,19 and a third study reported a saving of US\$1366 per participant in the intervention group by reducing sick days and productivity loss. ${ }^{22}$ Five trials reported no overall benefits in terms of cost savings. ${ }^{23,24,27,28,37}$

\section{Discussion}

This systematic review provides only very limited evidence that early multicomponent interventions are more effective than comparisons (both 'treatment as usual' and active interventions that did not meet our biopsychosocial criterion) in promoting return to work and reducing sickness absence among people with musculoskeletal pain. Lowquality evidence from the meta-analysis of four trials suggests that a stepped care approach is more effective than usual care in facilitating return to work. This is consistent with the suggestion that first-line interventions that include early access to treatment, reassurance about activity and work and/or workplace accommodation is sufficient for most workers, while more structured vocational rehabilitation is reserved for those who do not respond to conservative management. ${ }^{7}$ The analyses on sick leave data, which included 11 trials with a long-term follow-up period, did not find an effect for any of the five different categories of interventions above that of the comparison in reducing sick leave. Similarly, there was little or no difference in the effects of the intervention groupings on pain intensity and disability.

There was considerable variation in the duration of sick leave during the follow-up periods; in some cases, mean sick-leave data were skewed by the small number of participants who remained on long-term sick leave. It is not clear whether the variations in sick leave were related to the severity of symptoms at baseline or other factors, including the influence of the differing social protection systems within each jurisdiction. Considering the likelihood of recurring episodes of musculoskeletal pain, long-term follow-up periods are needed to provide information on the recurrence of absenteeism after the initial resumption of employment. Few studies in the current review reported on this, thus it was not possible to determine the extent to which further episodes of sick leave occurred. The effectiveness of interventions in preparing people to cope with reoccurrence of symptoms warrants further exploration.

It is not necessary for people to be pain free before they return to work, ${ }^{39}$ therefore it may not be surprising that the interventions made little or no difference to pain intensity compared with the comparison groups. There are, however, several possible explanations as to why we did not find more robust effects on our outcomes as methodological limitations may have biased effect estimates. First, in at least six studies the comparison groups were able to avail of a range of additional services, and it is possible that the benefits achieved by engaging in cointerventions reduced the likelihood of identifying differences in outcomes between the intervention and control groups. Second, variations in the delivery and acceptance of treatment may have substantially impacted outcomes. ${ }^{40}$ Treatment fidelity and participant compliance with the prescribed intervention were not reported consistently. Furthermore, as these were early interventions it is possible that some participants in both the intervention and comparison groups had low levels of symptom intensity at baseline and/or may have improved spontaneously as part of a natural disease course.

\section{Limitations of the review}

We conducted an extensive search, but it is possible that we failed to identify some eligible trials. We limited our search to publications since 1990, as it seems likely that clinical practice prior to this date was unlikely to meet our inclusion criteria. In addition, we omitted a number of potentially eligible studies from the review as we were unable to make contact with authors to confirm that trials met our inclusion criteria; the possibility that their 
inclusion may have changed our conclusions cannot be ruled out. This raises some important issues regarding the need for consistency of reporting, including for example, sufficiently detailed protocols and procedures, some agreement on defining terms such as return to work and a core set of outcomes. $^{41}$

While the current review does have some overlap with previous effectiveness reviews, ${ }^{9,10,39}$ our focus on early multicomponent interventions offered an opportunity to identify an optimal treatment approach soon after the start of difficulties in remaining at work. The inclusion criteria for the review were stringent in terms of employment and sick leave status, yet our relatively open definition of 'biopsychosocial' interventions resulted in considerable variation in the active components included in the trials. We based decisions as to which studies could be pooled for analyses, on a determination of sufficient clinical homogeneity. However, these subjective decisions are open to debate and the magnitude of effects of the individual studies may be different from the summary effect of the meta-analyses. An alternative approach would have been to specify the type of intervention to be included more tightly; however, there are some indications from our own experience and other reviews that further restrictions would have identified few studies sufficiently similar for analysis. , $^{8,42,43}$

A further limitation relates to the impact of combining data from participants with a range of musculoskeletal conditions. While more than a half of the included studies recruited patients with a single diagnosis of back pain $(n=13)$, we were unable to conduct any subgroup analyses in relation to diagnoses owing to an insufficient number of trials within each of our intervention categories. There is, however, some evidence that the same general principles for effective return to work strategies apply across the most common musculoskeletal conditions. ${ }^{7}$ Finally, we were also unable to conduct the other planned subgroup analyses, including baseline symptom severity, and the effects of age and gender because of the low number of trials within each intervention category.

\section{Implications}

Multicomponent interventions can be costly both in terms of money and time commitments. ${ }^{10} \mathrm{~A}$ stepped approach that introduces more complex interventions only for those who do not respond to conservative management may help to limit the use of more expensive components. Some uncertainty remains as to the optimum time for intervention, as it has been suggested that enrolling in an intervention too soon may delay the natural progression to return to work. ${ }^{11}$ Thus, the challenge is to identify and target those who are at risk of chronicity and disability, one promising approach is to screen for risk factors linked to delayed recovery and return to work as this may help to identify those workers who would benefit most from early intervention. ${ }^{6,44}$

Given the diversity of interventions included in this review, some caution in interpretation and application of the findings is warranted. The included trials were conducted across seven different countries with differing health services and social security systems. Variation also existed in the components constituting usual care for the control/comparison groups. These possible confounds have been acknowledged in other reviews. For example, a review of the effectiveness of multidisciplinary interventions on return to work for low back pain reported a clinically relevant effect only when the meta-analysis was limited to studies conducted in Scandinavia - that is, countries with similar labour markets, unemployment rates and insurance systems. ${ }^{42}$

\section{Clinical messages}

- There is still uncertainty regarding the effectiveness of early multicomponent interventions owing to clinical heterogeneity and varying health and social insurance systems.

- The need to identify the patients who are most likely to benefit and to establish the active components that promote work participation in this population remains. 


\section{Acknowledgements}

We wish to acknowledge the contributions of Roisin Breen (Royal College of Physicians in Ireland) and Aisling Brennan (Physiotherapy Services, AMNCH Dublin Ireland) in developing the inclusion criteria for the review, and Donal O'Neill (Professor of Economics Maynooth University) for his advice regarding the economic evaluation of included studies.

\section{Declaration of conflicting interest}

The author(s) declared no potential conflicts of interest with respect to the research, authorship, and/or publication of this article.

\section{Funding}

The author(s) disclosed receipt of the following financial support for the research, authorship, and/or publication of this article: This research was funded by the Health Research Board [RCQPS-2014-2].

\section{References}

1. Walker-Bone $\mathrm{K}$ and Cooper $\mathrm{C}$. Hard work never hurt anyone - or did it? A review of occupational associations with soft tissue musculoskeletal disorders of the neck and upper limb. Ann Rheum Dis 2005: 64(8): 1112-1117.

2. Bevan S. Back to work: Exploring the benefits of early interventions which help people with chronic illness remain in work, fit for work Europe. London: The Work Foundation, 2015.

3. Bevan S. Reducing temporary work absence through early intervention: The case of MSDs in the EU. London: The Work Foundation, 2013.

4. Waddell G and Aylward M. The scientific and conceptual basis of incapacity benefits. London: TSO, 2005.

5. Iles RA, Wyatt M and Pransky G. Multi-faceted case management: Reducing compensation costs of musculoskeletal work injuries in Australia. J Occup Rehabil 2012; 22: 478-488.

6. Briand C, Durand M-J, St-Arnaud L, et al. How well do return-to-work interventions for musculoskeletal conditions address the multicausality of work disability? $J$ Occup Rehabil 2008; 18: 207-217.

7. Waddell G, Burton AK and Kendall NAS. Vocational rehabilitation: What works for whom and when? Report for the Vocational Rehabilitation Task Group. London: TSO, 2008.

8. Palmer KT, Harris EC, Linaker C, et al. Effectiveness of community- and workplace-based interventions to manage musculoskeletal-related sickness absence and job loss: A systematic review. Rheumatology 2012; 51: 230-242.

9. Schandelmaier S, Ebrahim S, Burkhardt SCA, et al. Return to work coordination programmes for work disability: A meta-analysis of randomised controlled trials. PLoS ONE 2012; 7(11): e49760.
10. Kamper SJ, Apeldoorn AT, Chiarotto A, et al. Multidisciplinary biopsychosocial rehabilitation for chronic low back pain: Cochrane systematic review and meta-analysis. BMJ 2015; 350: h444.

11. van Duijn M, Ejkemans MJ, Koes BW, et al. The effects of timing on the cost-effectiveness of interventions for workers on sick leave due to low back pain. Occup Environ Med 2010; 67: 744-750.

12. Waddell G and Burton AK. Concepts of rehabilitation for the management of common health problems. London: TSO, 2004.

13. Buck R, Wynne-Jones G, Varnava A, et al. Working with musculoskeletal pain. Reviews in Pain 2009; 3(1): 6-10.

14. Macfarlane GJ, Pallewatte N, Paudyal P, et al. Evaluation of work-related psychosocial factors and regional musculoskeletal pain: Results from a EULAR task force. Ann Rheum Dis 2009; 68: 885-891

15. Liberati A, Altman DG, Tetzlaff J, et al. The PRISMA statement for reporting systematic reviews and meta-analyses of studies that evaluate health care interventions: Explanation and elaboration. PLoS Med 2009; 6(7): e10000100.

16. Guzmán J, Esmail R, Karjalainen, et al. Multidisciplinary bio-psycho-social rehabilitation for chronic low back pain. The Cochrane Database of Systematic Reviews 2002; Issue 1: Art. No.: CD000963.

17. Higgins JPT and Green S (eds) Cochrane handbook for systematic reviews of interventions 5.10 (updated March 2011) The Cochrane Collaboration 2011. Available from www.cochrane-handbook.org (accessed 12 July 2016).

18. Review Manager (RevMan) [Computer program]. Version 5.3. Copenhagen: The Nordic Cochrane Centre, The Cochrane Collaboration, 2014.

19. Abásolo L, Blanco M, Bachiller J, et al. A health system program to reduce work disability related to musculoskeletal disorders. Ann Intern Med 2005; 143: 404-414.

20. Heymans MW, de Vet HCW and Bongers PM. The effectiveness of high-intensity versus low-intensity back schools in an occupational setting. A pragmatic randomised controlled trial. Spine 2006; 10: 1075-1082.

21. Leclaire R, Esdaile JM, Suissa S, et al. Back school in a first episode of compensated acute low back pain: A clinical trial to assess efficacy and prevent relapse. Arch Phys Med Rehabil 1996; 77: 673-679.

22. Bültmann U, Sherson D, Olsen J, et al. Coordinated and tailored work rehabilitation: A randomised controlled trial with economic evaluation undertaken with workers on sick leave due to musculoskeletal disorders. J Occup Rehabil 2009; 19: 81-93.

23. Jensen C, Jensen OK, Christiansen DH, et al. Oneyear follow-up in employees sick-listed because of low back pain. Randomised clinical trial comparing multidisciplinary and brief intervention. Spine 2011; 15: 1180-1189.

24. Schultz IZ, Crook JM, Berkowitz J, et al. Early intervention with compensated lower back-injured workers at risk for work disability: Fixed versus flexible approach. Pschol Inj and Law 2013; 6: 258-276. 
25. Stapelfeldt CM, Christiansen DH, Jensen OK, et al. Subgroup analyses on return to work in sick-listed employees with low back pain in a randomised trial comparing brief and multidisciplinary intervention. $B M C$ Musculoskeletal Disorders 2011; 12(112). DOI: 10.1186/ 1471-2474-12-112.

26. Andersen LN, Juul-Kristensen B, Roessler KK, et al. Efficacy of 'tailored physical activity' on reducing sickness absence among health care workers: A 3-month randomised controlled trial. Manual Therapy 2015; 20: 666-671.

27. Bernaards CM, Bosmans JE, Hildebrandt VH, et al. The cost-effectiveness of a lifestyle physical activity intervention in addition to a work style intervention on recovery from neck and upper limb symptoms and pain reduction in computer workers. Occup Environ Med 2011; 68: 265-272.

28. Karjalainen K, Malmivaara A, Pohjolainen T, et al. Miniintervention for subacute low back pain. A randomised controlled trial. Spine 2003; 6: 533-541.

29. Rantonen J, Luoto S, Vehtari A, et al. The effectiveness of two active interventions compared to self-care advice in employees with non-acute low back symptoms: A randomised controlled trial with a 4-year follow-up in the occupational health setting. Occup Environ Med 2012; 69: 12-20.

30. Hutting N, Staal JB, Engels JA, et al. Effect evaluation of a self-management programme for employees with complaints of the arm, neck or shoulder: A randomised controlled trial. Occup Environ Med. Epub ahead of print 10 September 2015. DOI: 10.1136/oemed-2015-103089.

31. Jensen LD, Maribo T, Schiøttz-Christensen B, et al. Counselling low-back-pain patients in secondary healthcare: A randomised trial addressing experienced workplace barriers and physical activity. Occup Environ Med 2012; 69: 21-28.

32. Linton SJ, Hellsing A-L and Larsson I. Bridging the gap: Support groups do not enhance long-term outcome in chronic back pain. Clin J Pain 1997; 13: 221-228.

33. Linton SJ, Boersma K, Jansson M, et al. The effects of cognitive-behavioural and physical therapy preventive interventions on pain-related sick leave. A randomised controlled trial. Clin J Pain 2005; 21(2): 109-119.
34. Linton SJ, Boersma K, Traczyk M, et al. Early workplace communication and problem solving to prevent back disability: Results of a randomised controlled trial among high-risk workers and their supervisors. J Occup Rehabil 2016; 26: 150-159.

35. Schiltenwolf M, Buchner M, Heindl B, et al. Comparison of a biopsychosocial therapy (BT) with a conventional biomedical therapy (MT) of subacute low back pain in the first episode of sick leave: A randomised controlled trial. Eur Spine J 2006; 15: 1083-1092.

36. Leon L, Jover JA, Candelas G, et al. Effectiveness of an early cognitive-behavioral treatment in patients with work disability due to musculoskeletal disorders. Arthritis Rheum 2009; 61(7): 996-1003.

37. Loisel P, Abenhaim L, Durand P, et al. A populationbased, randomised clinical trial on back pain management. Spine 1997; 22(24): 2911-2918.

38. Anema JR, Steenstra IA, Bongers PM, et al. Multidisciplinary rehabilitation for subacute low back pain: Graded activity or workplace intervention or both? Spine 2007; 32(3): 291-298.

39. Nguyen $T$ and Randolph DC. Nonspecific low back pain and return to work. Am Fam Physician 2007; 76(10): 1497-1502.

40. Gauthier N, Sullivan MJL, Adams H, et al. Investigating risk factors for chronicity: The importance of distinguishing between return-to-work status and self-report measures of disability. JOEM 2006; 48(3): 312-318.

41. Herbert RD and Bö K. Analysis of quality of interventions in systematic reviews. BMJ 2005; 331: 507-509.

42. Norlund A, Ropponen A and Alexanderson K. Multidisciplinary interventions: Review of studies of return to work after rehabilitation for low back pain. J Rehabil Med 2009; 41: 115-121.

43. van Vilsteren M, van Oostrum $\mathrm{SH}$, de Vet $\mathrm{HCW}$, et al. Workplace interventions to prevent work disability in workers on sick leave. Cochrane Database of Systematic Reviews 2015; Issue 10: Art No.: CD006955.

44. Linton SJ and Shaw WS. Impact of psychological factors in the experience of pain. Phys Ther 2011; 91(5): $700-711$. 